\title{
Core-collapse Supernovae
}

\author{
W. Raphael Hix ${ }^{* a b}$, Eric J. Lentz ${ }^{b}$, Mark Baird ${ }^{c}$ Austin Chertkow $^{b}$, Ching-Tsai Lee $^{b}$, \\ John Blondin $^{c}$, Stephen Bruenn ${ }^{d}$, O.E. Bronson Messer ${ }^{e b}$, Anthony Mezzacappa ${ }^{a b \dagger}$ \\ ${ }^{a}$ Physics Division, Oak Ridge National Laboratory \\ Oak Ridge TN 37831-6354 \\ ${ }^{b}$ Department of Physics and Astronomy, University of Tennessee \\ Knoxville, TN 37996-1200 \\ ${ }^{c}$ Reactor \& Nuclear Systems Division, Oak Ridge National Laboratory \\ Oak Ridge TN 37831-6003 \\ ${ }^{d}$ Department of Physics, North Carolina State University, \\ Raleigh, NC 27695-8202 USA \\ e Department of Physics, Florida Atlantic University, \\ 777 W Glades Road, Boca Raton, FL 33431-0991 USA \\ ${ }^{f}$ Center for Computational Sciences, Oak Ridge National Laboratory \\ Oak Ridge TN 37831-6008
}

\begin{abstract}
Marking the inevitable death of a massive star, and the birth of a neutron star or black hole, core-collapse supernovae bring together physics at a wide range in spatial scales, from kilometersized hydrodynamic motions (growing to gigameter scale) down to femtometer scale nuclear reactions. Carrying $10^{51}$ ergs of kinetic energy and a rich-mix of newly synthesized atomic nuclei, core-collapse supernovae are the preeminent foundries of the nuclear species which make up ourselves and our solar system. We will discuss our emerging understanding of the convectively unstable, neutrino-driven explosion mechanism, based on increasingly realistic neutrino-radiation hydrodynamic simulations that include progressively better nuclear and particle physics. Recent multi-dimensional models with spectral neutrino transport from several research groups, which slowly develop successful explosions for a range of progenitors, have motivated changes in our understanding of the neutrino reheating mechanism. In a similar fashion, improvements in nuclear physics, most notably explorations of weak interactions on nuclei and the nuclear equation of state, continue to refine our understanding of how supernovae explode. Recent progress on both the macroscopic and microscopic effects that affect core-collapse supernovae are discussed.
\end{abstract}

VI European Summer School on Experimental Nuclear Astrophysics, ENAS 6

September 18-27, 2011

Acireale Italy

\footnotetext{
${ }^{*}$ Speaker.

$\dagger$ This work supported by grants from the NASA Astrophysics Theory Program (NNH11AQ72I) and the NSF PetaApps, Nuclear Physics and Stellar Astrophysics Programs (OCI-0749242,AST-0653376) and by the Department of Energy Offices of Nuclear Physics and Advanced Scientific Computing Research.
} 


\section{Introduction}

As "new stars" that make their appearance in the sky, supernovae have long been a source of wonderment, reported throughout at least 2 millennia of recorded history. With a visual display that can compete in brightness with its entire host galaxy, supernovae are among the most energetic astrophysical phenomena. The ejecta of a supernova delivers $10^{51} \mathrm{ergs}$ of kinetic energy and a rich mix of recently synthesized elements into the interstellar medium. These explosions are the dominant foundry of the heavy elements that have been formed since the Big Bang. They represent a major source of heat in the ISM as well as a potential trigger for star formation [see, e.g., 1, 2]. Observationally, these explosions are categorized into two types, Type I and Type II, based on the presence of hydrogen in their spectra soon after the explosion. Numerous sub-types are based on the shape of the light curve and the presence or shape of other spectral lines [for more discussion, see, e.g., 3, 4]. While Type Ia supernovae, classified by their lack of hydrogen lines but the presence of strong Si II lines in their early-time spectra, are believed to be powered by the thermonuclear ignition of white dwarves [see, e.g., 5-8] in binary systems, the remaining supernova types and subtypes are thought to result from the deaths of individual massive stars. The wide variations between types and sub-types come not from different explosion mechanisms, but from differences in the stellar envelope as a result of stellar evolution and mass loss.

To understand the deaths of massive stars, we must begin long before the explosions that mark these deaths and distribute these materials into the interstellar medium. From its initial contraction to form a hydrogen-burning main sequence star, nuclear burning in the stellar core, and in shells surrounding the core, provide the energy to resist gravitational contraction and light the star. Once a star exhausts the hydrogen in its core, the now helium-rich core contracts. This ignites hydrogen in a shell lying atop the core, boosting the luminosity of the star and driving the stellar surface outward to become a Red Giant. Continued contraction of the core is halted by the ignition of helium burning. This evolution sets the pattern for the star's life, with the ash of each stage becoming the fuel for its successor.

For stars below a critical mass -8 solar masses (hereafter denoted $M_{\odot}$ ) is commonly adopted [9], though the number may be as small as $6 M_{\odot}[10]$ - degeneracy in the Carbon-Oxygen (CO) core prevents the ignition of carbon burning. These low-mass stars end their lives as cooling $\mathrm{CO}$ white dwarves with masses less than $1.1 M_{\odot}$, surrounded by the envelope they ejected in their Asymptotic Giant Branch (AGB) stellar winds. For much more massive stars, from perhaps 11 $M_{\odot}$ up to roughly $100 M_{\odot}$ [11], hydrostatic carbon, neon, oxygen, and silicon burning leave a core composed of iron, cobalt, nickel, and neighboring species, commonly referred to as the iron-peak nuclei because they populate a peak in the solar system abundance distribution. Once this core grows beyond the maximum stable mass for a system supported by electron degeneracy pressure (the so-called Chandrasaekhar mass), it collapses. For stars in the lower portion of this mass range, by means we will discuss momentarily, the collapse produces a neutron star and a shock wave that disrupts the stellar envelope, leading to a supernova. In the higher mass region, a black hole forms, preventing the shock wave from being sufficiently energized to disrupt the stellar envelope, and the supernova fails. However, if the progenitor star is rapidly rotating, an alternate mechanism, the collapsar mechanism [12] - driven by an accretion disk surrounding the black hole and producing jets along the rotational axis - may produce a peculiar, hyper-energetic supernova explosion 
(with explosion energies as much as an order of magnitude larger) and an associated gamma-ray burst. Stars in the intermediate range, above $8 M_{\odot}$ but below $11 M_{\odot}[13]$, successfully ignite carbon and neon burning, but under degenerate conditions, leading to massive Oxygen-Neon-Magnesium $(\mathrm{ONeMg})$ white dwarves. Near the upper boarder of this range, above perhaps $10.5 M_{\odot}[14]$, models suggest that shell burning causes the mass of the ONeMg core to increase beyond the stable Chandrasaekhar mass before the star loses its envelope. Here too, the core collapses, producing a supernova [15]. Two critical distinctions differentiate the supernovae of these ONeMg cores, also known as electron-capture supernovae (ECSN), from their iron-core brethren: (1) a collapse driven by electron capture on ${ }^{23} \mathrm{Na},{ }^{20} \mathrm{Ne}$, and ${ }^{24} \mathrm{Mg}$ rather than on iron-peak nuclei and (2) a low-density helium shell surrounding the core in the former case, in place of dense layers of silicon and oxygen in the latter.

\section{Core-Collapse Supernovae}

The collapse of the stellar core, whether composed initially of iron or oxygen-neon-magnesium, proceeds until super-nuclear densities, larger than the densities inside atomic nuclei, are reached. The inner core, which has collapsed homologously, becomes incompressible under these extremes, bounces, and, acting like a piston, launches a shock wave into the outer stellar core, which is infalling supersonically. This shock wave will ultimately propagate through the stellar layers beyond the core and disrupt the star in a supernova explosion. However, the shock initially stalls in the outer core, having lost energy to escaping neutrinos and the dissociation of the heavy nuclei into free nucleons and $\alpha$-particles as it plows through the stellar core. How the shock is revived is the central question in core-collapse supernova theory [for more details, see, e.g., 16, 17].

After core bounce, more than $10^{53}$ ergs of energy in the form of neutrinos and antineutrinos of all three flavors (electron, muon, and tau) is released from the newly formed proto-neutron star (PNS) at the center of the explosion, 100 times more energy than the observed kinetic energy of the supernova explosion. Past simulations $[18,19]$ demonstrate that energy in the form of neutrinos emerging from the PNS can be deposited behind the shock and may revive it. However, while a prodigious amount of neutrino energy emerges from the PNS, the neutrinos are weakly coupled to the material directly below the shock. The neutrino heating, which occurs primarily by the absorption of electron neutrinos and antineutrinos on the dissociation-liberated free nucleons, is very sensitive to the spectral distribution of neutrinos [20-23] and direction of propagation (specified uniquely by two angles), at any given spatial point behind the shock [24, 25]. In turn, this ultimately requires spectral, multi-angle (Boltzmann) neutrino transport in order to accurately compute the neutrino distributions in this region in energy and angle. This renders neutrino transport in core-collapse supernovae a six dimensional (space plus neutrino energy and angles) problem. On current supercomputer architectures, an approach to this ultimate objective must be staged, beginning with spatially $3 \mathrm{D}$, spectral moments models of the neutrino radiation field and progressing eventually to spectral, multi-angle Boltzmann models. In these moments models, the neutrino distributions in angle are approximately represented by the lowest order angular moments of the neutrino distribution function: the neutrino energy density and three momentum densities (one for each spatial dimension), all as a function of neutrino energy. While this is an approximation to the 
full Boltzmann treatment, moments models can be quite sophisticated and can describe well what would be obtained if the full Boltzmann equation were solved.

The neutrino heating may be aided by fluid instabilities (e.g., convection) in the PNS [26-30], which may boost the luminosity of this central neutrino bulb. Convection occurring directly beneath the shock fundamentally alters the nature of neutrino shock reheating [21, 30-34] relative to the spherically symmetric case, allowing simultaneous downward flows that fuel the neutrino luminosities by accretion onto the PNS and upward flows that bring energy to the shock. A recently discovered multidimensional instability of the shock wave itself, the Standing Accretion Shock Instability (SASI; [35]), dramatically alters the shock and explosion dynamics [35-38]. Centrifugal effects in a rotating stellar core $[30,33]$, and other rotational effects, can change supernova dynamics quantitatively and perhaps qualitatively. Stellar core magnetic fields, increased by compression during collapse, convection (e.g., via a dynamo), and rotation (through wrapping and shear; in the latter case the magnetorotational instability may occur and, if so, would dominate the field evolution), may also play a significant role in driving, and perhaps collimating, at least a subset of core-collapse supernova explosions where rapid rotation is present [39-41]. Nuclear burning must also be included, as the energy released by burning in the compressed and heated material near the shock wave helps to power the explosion. Finally, the PNS is an extremely dense, compact object, and its gravitational field is not well described by Newtonian gravity. Rather, General Relativity (GR) is required. It is, after all, the release of gravitational binding energy as the core collapses and the PNS forms that provides the energy in neutrinos, which we believe ultimately powers the expulsion of the stellar envelope. Thus, GR is fundamental to the energetics of core-collapse supernovae.

Recent years have seen notable progress toward ascertaining the core-collapse supernova explosion mechanism. Groups worldwide using three independent codes (CHIMERA [42], VERTEX [43], and Zeus+IDSA [44]) have now reported neutrino-driven explosions using spectral neutrino transport, across a range of stellar progenitor masses from 11 to $25 \mathrm{M}_{\odot}$. In all these cases, the SASI couples with neutrino shock reheating to power the explosions and thus plays a central role. In contrast, Burrows and collaborators [37, 45], using the Vulcan/2D code have reported successful explosions in which the shock is revived not by neutrino reheating, which is insufficient in their simulations, but by acoustic waves excited by the SASI-induced aspherical accretion stream onto the PNS. Unfortunately, the above encouraging results were obtained in 2D simulations while the hydrodynamic instabilities are manifestly three dimensional. Three dimensional models with all essential physics are crucial and now appear on the horizon. However, at present such models are so extremely computationally expensive that only a handful are likely to be run in the next 5 years.

\section{Essential Ingredients in Core-Collapse Supernovae}

From the discussion in Section 凹, many of the forms of physics operating in core-collapse supernovae are immediately clear; gravity, fluid dynamics, neutrino-matter interactions, neutrino transport, thermonuclear kinetics. However, the question of what physics ingredients are truly essential to the core-collapse supernova mechanism, and at what level of detail, is a subject of great debate among the modelers of these events. This question is made important by the increased computational cost of improved physical fidelity, leading modelers to adopt the least expensive im- 
plementation of each ingredient that they believe has sufficient fidelity. In the following subsections we will highlight several of these ingredients, starting with the nuclear physics of interest.

\subsection{Nuclear Physics and the Central Engine}

The impact of nuclear physics on core-collapse supernovae after the formation of the supernova shock is limited by the shock's dissociation of heavy nuclei into free nucleons. Beyond the core collapse phase, only deep in the core, where the nucleons form nuclear matter, and in the outer regions where the shock is not strong enough to fully dissociate nuclei, does nuclear physics play a large role. Thus our interest in nuclear physics is focused on the behavior of nuclei at very high densities and those reaction rates that can contribute during core collapse or to the nucleosynthesis caused by the shock's outward propagation, which we will discuss in Section $⿴$.

\subsubsection{Nuclear Equation of State}

The dominance of the maximally bound, iron-peak nuclei in the iron core is the result of thermodynamic equilibrium for strong and electromagnetic nuclear reactions, termed Nuclear Statistical Equilibrium (NSE). During collapse, the increasing density and neutronization causes NSE to favor heavier, more neutron-rich nuclei. This leads eventually to a composition dominated by exotic columnar and planar nuclear states [46] and ultimately, when the core reaches densities similar to those of the nucleons in a nucleus, nuclear matter. The development of NSE makes the nuclear composition a function of the thermodynamic state and electron fraction $\left(\mathrm{Y}_{e}\right)$, allowing the composition to be evolved as part of the equation of state, unlike concentric layers of successively lighter elements that lie above the iron core, whose evolution must be followed with a nuclear reaction network. Ultimately, all of these phases of baryonic matter must be included in the supernova equation of state $(\mathrm{EoS})$. In general, the $\mathrm{EoS}$ is a thermodynamic relation between the pressure, temperature and internal energy, providing a closure to the hydrodynamic equations. For stellar matter, contributions from electrons/positrons, photons and atomic nuclei all must be considered.

The thermodynamic state and composition of the core naturally has a profound impact on the hydrodynamic evolution and neutrino radiation transport in core-collapse supernovae. For example, the matter pressure, determined by the thermodynamic state, causes the bounce and drives much of the hydrodynamic motion, while the matter composition interacts with the neutrino opacities, determining how effectively energy is transferred from the neutrino radiation field to the matter. For these reasons, considerable effort has been invested in supernova equations of state. In addition to thermodynamic variables like pressure, internal energy and entropy, these EoSs provide nuclear compositional data, usually in the form of the mass fractions of protons, neutrons, $\alpha$-particles and an average heavy nucleus, along with the atomic number and mass of this average heavy nucleus.

The equation of state of Lattimer \& Swesty [47] (LS) has long been the staple EoS for supernova simulations. It is based on a compressible liquid drop model, similar to that of Lamb et al. [48], but in a lightweight form suitable for calculation within a supernova model. The nuclear composition for non-uniform nuclear matter is calculated in the Wigner-Seitz approximation of a heavy nucleus in a vapor of nucleons and $\alpha$-particles. The LS EoS assumes a nuclear saturation density $\left(\rho_{s}\right)$ of $2.59 \times 10^{14} \mathrm{~g} \mathrm{~cm}^{-3}$, a symmetry energy $\left(E_{s y m}\right)$ of $29.3 \mathrm{MeV}$ and allows a selection of the nuclear incompressibility $\left(\kappa_{s}\right)$. For consistency with our earlier work, the simulations described in this manuscript use $\kappa_{s}=180 \mathrm{MeV}$, but simulations using a more realistic $\kappa_{s}=220 \mathrm{MeV}$ are similar. 


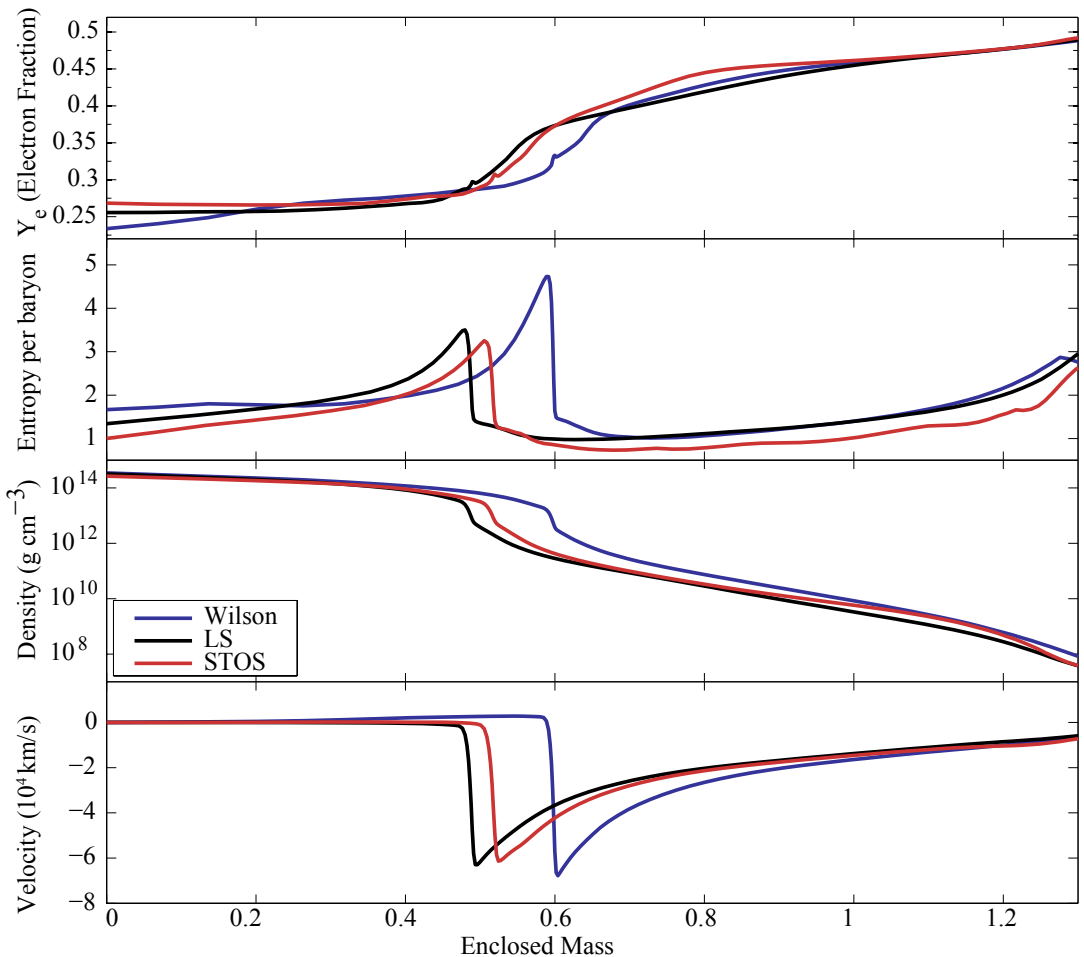

Figure 1: Effects of the equation of state at bounce for a $15 M_{\odot}$ progenitor. The black, red and blue lines illustrate results from simulations using the EoS of Lattimer and Swesty [47], Shen et al. [49], and Wilson and Mathews [51], respectively.

The equation of state of Shen, Toki, Oyamatsu \& Sumiyoshi [49] (STOS) is based on the relativistic mean field theory. It is constructed assuming $\rho_{s}=2.43 \times 10^{14} \mathrm{~g} \mathrm{~cm}^{-3}, E_{s y m}=36.9 \mathrm{MeV}$ and $\kappa_{s}=281 \mathrm{MeV}$. Thomas-Fermi calculations [50] provide the nuclear composition for non-uniform nuclear matter, also using the Wigner-Seitz approximation. The equation of state of Wilson and his collaborators is described most fully in Wilson and Mathews [51] (see also [52-54]). For supranuclear matter, the empirical prescription of Baron et al. [55] is used with $\rho_{s}=2.66 \times 10^{14} \mathrm{~g} \mathrm{~cm}^{-3}$, $\kappa_{s}=200 \mathrm{MeV}$ and the supranuclear adiabatic index, $\gamma_{s},=2.75$. These choices for $\rho_{s}, \kappa_{s}$ and $\gamma_{s}$, as well as the form of the symmetry energy $E_{s y m}=16\left(1-2 \mathrm{Y}_{e}\right)^{2}\left(\rho / \rho_{s}\right)\left(1+4.5 /\left(1+4 \rho / \rho_{s}\right)\right) \mathrm{MeV}$, were informed by relativistic Brueckner-Hartree-Fock calculations [56]. The Wilson EoS also includes the effects of pion production at high density, using the model of Friedman et al. [57]. This model is constrained by comparision between experimental measurements and simulations of pion production in heavy ion collisions [54]. The identity of the average heavy nucleus is calculated from a density and electron fraction dependent analytic formula [51].

Recent years have seen a renaissance in nuclear Equations of State valid for core-collapse supernovae, which, unlike the cold neutron star case, require the effects of non-zero temperature. These include new EoS by Hempel and Schaffner-Bielich [58, hereafter HS], Shen et al. [59, hereafter SHT], and Shen et al. [60, hereafter SHO]. SHT and SHO are Hartree approaches in which the density profiles are self-consistently calculated. HS is based on an ensemble of nuclei in nuclear statistical equilibrium (NSE). All of these approaches have different benefits and drawbacks.

In order to illustrate the impact that the EoS can have on a core-collapse supernova simulation, 


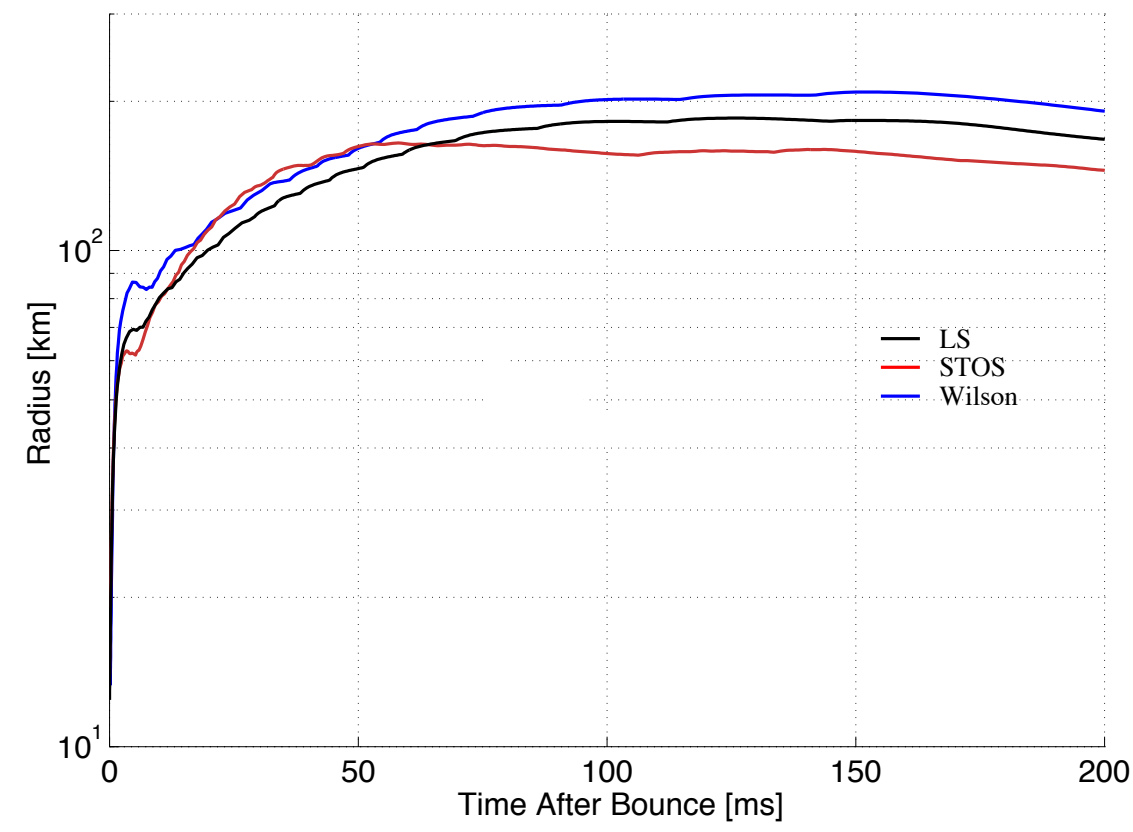

Figure 2: Impact of equation of state variations on the radius of the supernova shock as a function of time. The black, red and blue lines illustrate results from simulations using the the EoS of Lattimer and Swesty [47], Shen et al. [49], and Wilson and Mathews [51], respectively.

we compared three EoS; LS, STOS and Wilson. Full simulations were run with each of the three EoSs, using Agile-BOLTZTRAN [61]. These simulations include General Relativity (GR), which is important for a nuclear EoS comparison since the inclusion of GR results in roughly $30 \%$ higher central densities thus better exercising the supranuclear portion of the EoS. To facilitate comparison with the results of Hix et al. [62], neutrino opacities consistent with those simulation were used here, those from Bruenn [63] with the Langanke-Martinez-Pindeo-Sampaio-Hix (LMSH) nuclear electron prescription [see 62,64]. Figure $\square$ shows the conditions at bounce for the three EoS as a function of enclosed mass. The Wilson EoS results in a much larger homologous core $\left(0.58 M_{\odot}\right)$ than either STOS $\left(0.52 M_{\odot}\right)$ or LS $\left(0.48 M_{\odot}\right)$ cases. For much of the collapse, $\mathrm{Y}_{e}$ for the Wilson cases is higher than the others, resulting in the larger core. At bounce, this effect is still apparent in the outer part of the inner core $\left(0.2-0.5 \mathrm{M}_{\odot}\right)$, though a sharp gradient in $\mathrm{Y}_{e}$ develops in the Wilson EoS case over the last few ms before bounce in the regions with density above $2 \times 10^{14} \mathrm{~g} \mathrm{~cm}^{-3}$, where the pion contribution becomes significant in this EoS. This decrease in the $\mathrm{Y}_{e}$ is consistent with previous results [53] and is caused by the impact of the pions on the free nucleon chemical potentials. The differences in $\mathrm{Y}_{e}$ at lower density between the three EoSs result from differences in the composition. For example, the largest difference in heavy nucleus mass fractions between the STOS and LS EoS results in a noticeable rise in $\mathrm{Y}_{e}$ around $0.8 M_{\odot}$ in the STOS case. Likewise, the generally lower heavy nucleus mass fraction of the Wilson EoS results in less electron capture consistently throughout the star.

Figure $\square$ shows the time history of the shock over the $200 \mathrm{~ms}$ after bounce. Note, the small scale noise seen beyond $10 \mathrm{~ms}$ in Figure $\square$ results from the determination of the location of the shock in Agile-BOLtZTRAN. In simulations with the LS EoS $\left(\kappa_{s}=180 \mathrm{MeV}\right)$, the shock is launched, and reaches approximately $189 \mathrm{~km}$ (enclosed mass of $1.38 M_{\odot}$ ) from the central core 
before stalling $130 \mathrm{~ms}$ after bounce. In contrast, the shock in simulations using the STOS only reaches a distance of approximately $165 \mathrm{~km}\left(1.32 M_{\odot}\right)$ before stalling. This maximum also occurs much sooner (60 ms after bounce) than in the LS EoS model. With $\kappa_{s}=180 \mathrm{MeV}, \mathrm{LS}$ EoS is a relatively soft EoS and, as a consequence, stores a larger amount of energy in the PNS than a stiffer EoS, allowing the shock to travel further out before stalling due to energy loses from neutrino emission and the disassociation of heavy nuclei. The STOS EoS, with $\kappa_{s}=280 \mathrm{MeV}$, is stiffer than the LS EoS, so while the initial pressures are higher and thus the early progress of the shock more rapid, less energy is stored in compression of the PNS, even though the initial PNS mass in the STOS case is $8 \%$ larger than in the LS case. As a result the shock can not travel as far out from the central core before stalling. The shock in simulations with the Wilson EoS travels out to a distance of approximately $209 \mathrm{~km}\left(1.44 M_{\odot}\right)$ before stall, $\sim 20 \mathrm{~km}$ further than the LS EoS case, and $\sim 44 \mathrm{~km}$ further than the STOS EoS. Even though the Wilson EoS, with $\kappa_{s}=200 \mathrm{MeV}$, is nominally stiffer than the LS EoS, it provides more energy to the shock than the LS EoS does, likely as a consequence of both the high density pion contribution and the larger initial size of the PNS caused by reduced electron capture. Even $200 \mathrm{~ms}$ after bounce, by which time convective instabilities will have developed in the neutrino heated region, the shock's location, which marks the outer boundary of this unstable region, exhibits a significant $(\sim 20 \%)$ variation due to the EoS. This is a clear indication that the EoS is important to the physics of core collapse and bounce and its impact can extend even to times well after bounce.

\subsubsection{Electron Capture during Core Collapse}

The composition of the iron core of the star, as dictated by Nuclear Statistical Equilibrium (NSE), as well as the pressure, entropy and other thermodynamic quantities calculated within the Equation of State, are based on the temperature, baryon density and electron fraction. Evolution of the electron fraction is determined not just by the nuclear composition, as we discussed in the previous section, but also by the reaction rates for processes like electron and neutrino capture that can alter the neutronization of the matter. Bethe et al. [65] pointed out that, due to the low entropy of the stellar core and resulting dominance of heavy nuclei over free nucleons, electron capture processes on heavy nuclei would dominate the evolution of the electron fraction during the late stages of stellar evolution and stellar core collapse. In the iron core, this predominantly occurs via GamowTeller (GT) transitions changing protons in the $f_{7 / 2}$ level into neutrons in the $f_{5 / 2}$ level. However, it was soon realized that as core collapse proceeds, increasing neutronization and density lead to average neutron numbers $>40$, filling the neutron $f_{5 / 2}$ orbital and quenching further electron capture on heavy nuclei. Calculations using the independent particle model (IPM) showed that neither thermal excitations nor forbidden transitions substantially alleviated this blocking $[66,67]$. However, it is well known that the residual nuclear interaction (beyond the IPM) mixes the $f p$ and $g d s$ shells, for example, making the closed $g_{9 / 2}$ shell a magic number in stable nuclei $(N=50)$ rather than the closed $f p$ shell $(N=40)$. Full shell model diagonalization calculations remain impossible in this regime due to the large number of available levels in the combined $f p+g d s$ system [68]. Langanke et al. [69] developed a "hybrid" scheme, employing Shell Model Monte Carlo (SMMC) calculations of the temperature-dependent occupation of the various single-particle orbitals to serve as input to Random Phase Approximation (RPA) calculations for allowed and forbidden transitions to calculate the capture rate. This approach enabled Langanke et al. [64] to demonstrate that elec- 


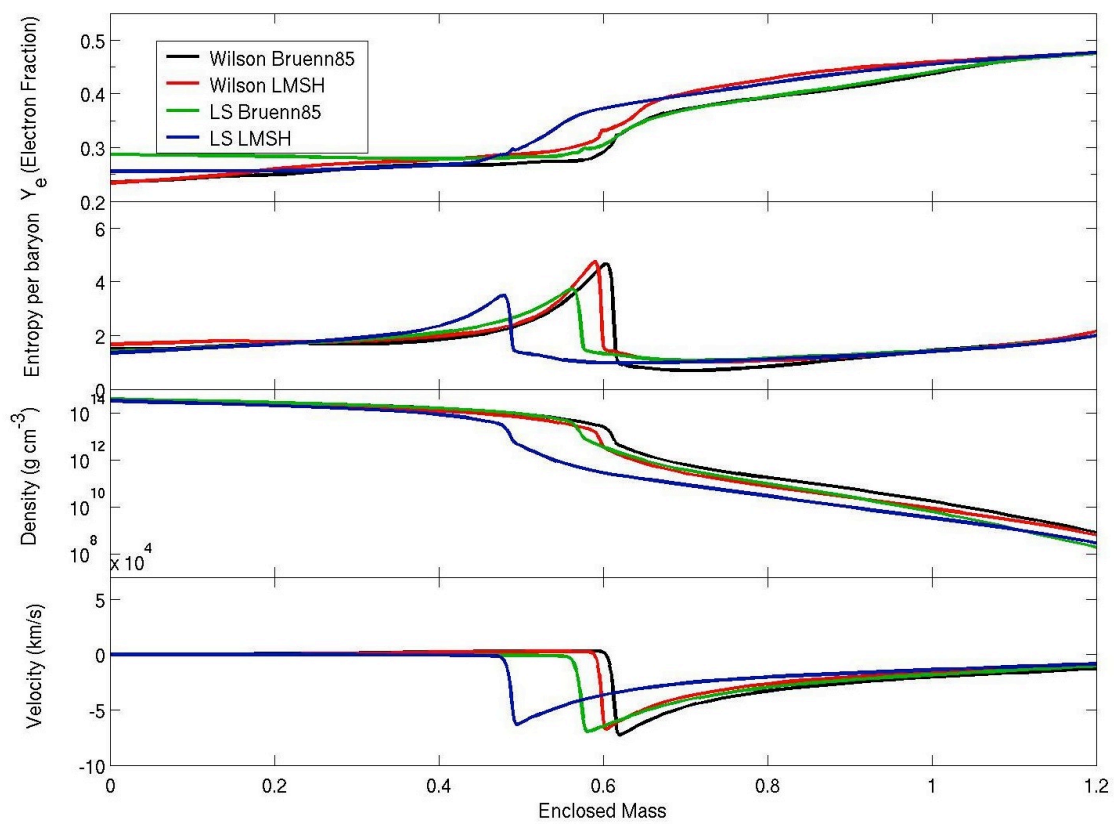

Figure 3: The combined effects of nuclear electron capture and the equation of state at bounce for a $15 M_{\odot}$ progenitor. The black and red lines illustrate results from simulations using the EoS of Wilson and Mathews [51], with [63] and modern nuclear electron capture, respectively. The green and blue lines illustrate the much largee effect of the transition from [63] and modern nuclear electron capture has when the EoS of Lattimer and Swesty [47] is used.

tron capture on heavy nuclei dominates the capture on protons throughout core collapse. While direct measurement of electron capture on these radioactive species is prohibitive, charge exchange reactions are being used to test the theoretical Gamow-Teller strength distribution underlying these rate calculations [see, e.g. 70, for discussion]. Juodagalvis et al. [71] have recently updated the compilation of theoretical nuclear electron capture rates needed for core-collapse supernovae.

As a result of the continuation of nuclear electron capture throughout collapse, Hix et al. [62] demonstrated that modern electron capture rates markedly reduced $(\sim 10 \%)$ the electron fraction in the interior of the PNS, resulting in an $\sim 20 \%$ reduction in the initial mass of the PNS, for a 15 solar mass progenitor using the LS EoS. This effect is complementary to those discussed in the preceding section, as the total electron capture rate depends on both the electron capture rates and the nuclear abundances provided by the EoS. Figure B demonstrates that the effect of the transition from IPM nuclear electron capture rates [63] to modern rates is much smaller for the Wilson EoS that for the LS EoS. While in the LS EoS case the initial mass of the PNS is reduced by nearly $0.09 M_{\odot}$, in the Wilson EoS case, it is reduced by slightly more than $0.01 M_{\odot}$. This is an excellent example of the interplay between different pieces of microscopic physics in the core-collapse supernova problem. Were the compositions provided by the as-yet-unknown true core-collapse supernova EoS to be more similar to those of Wilson than LS or STOS, the effects of modern nuclear electron capture could be more easily neglected. Lentz et al. [72] have recently shown that the softer neutrino spectrum from modern nuclear electron capture fills the low-energy neutrino spectrum without requiring neutrino-electron scattering. 


\subsection{Neutrino Transport Necessities}

As we discussed in Sect. \, forty years of computational study have revealed the need to model the neutrino spectra in at least moderate detail, dividing the neutrino spectrum into tens of energy bins. However other questions relevant to the cost of developing and using supernova codes remain, include the completeness of the GR implementation, which opacities to include, and the completeness of the neutrino transport in both dimension and relativity. Many of these questions can be addressed with numerically less expensive 1D simulation during the early epoch of SN evolution where spherical symmetry is a reasonable approximation.

To understand many of the general approximations made in the multi-D supernova codes, Lentz et al. [73] computed early phase 1D supernova models using Agile-BOLTZTRAN, by starting from a full-featured supernova model and stripped away major features to approximate the variation among the multi-dimensional supernova codes. Starting with a model with full GR hydrodynamics and transport and a modern neutrino opacity set, first the GR effects, then many of the opacities, and finally the velocity-dependent 'observer corrections' were removed. As we can see from the bounce

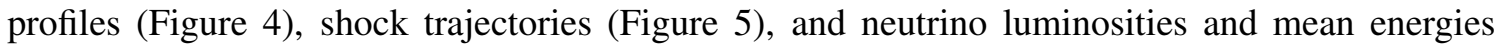
(Figure G) each of these broad changes has a noticeable impact on the conditions and evolution of the collapsed core. The starting point for these tests is a model (GR-FullOp, black lines) that includes a full treatment of general relativity in the hydrodynamics and transport and up-to-date neutrino opacities that include energy-exchanging scatterings on nucleons and electrons, nucleonnucleon bremsstrahlung, and the detailed LMSH EC table.
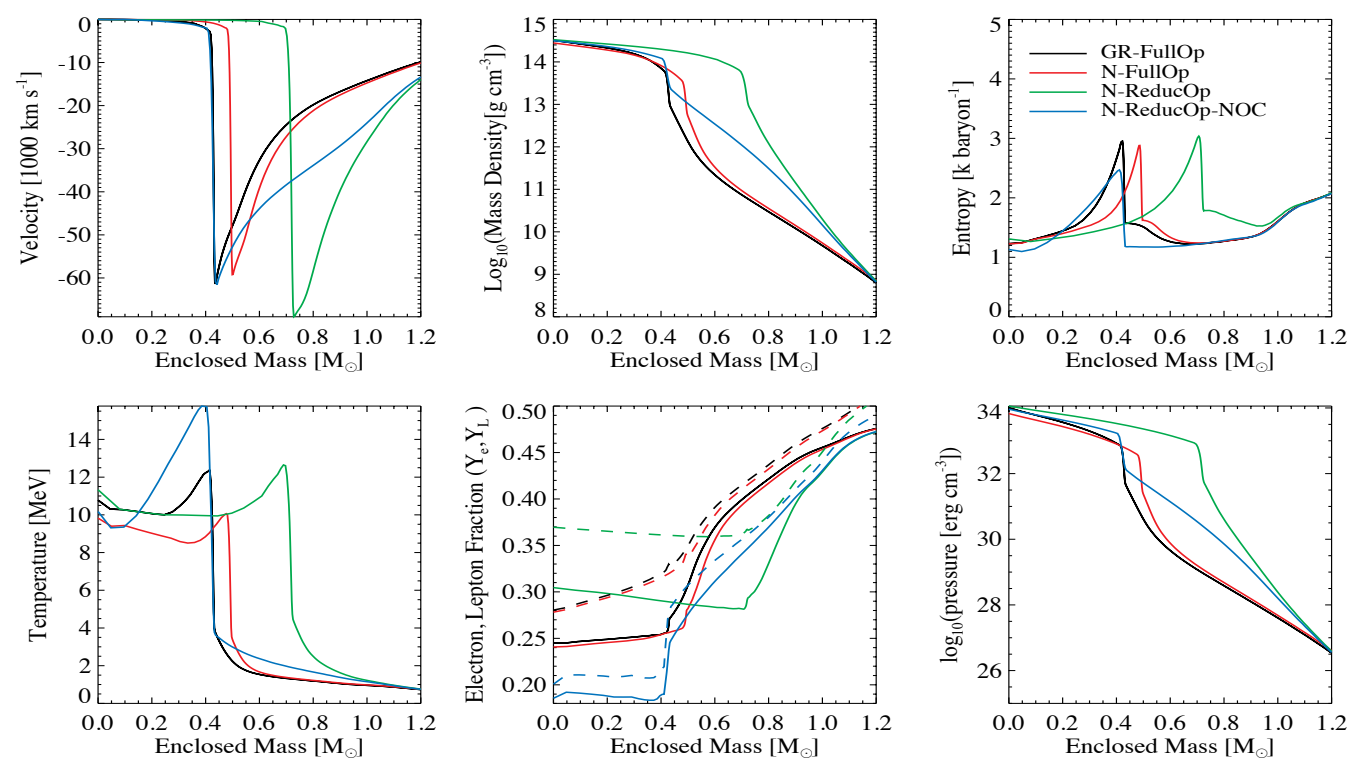

Figure 4: Properties of models at core bounce [73]. The models are: general relativistic gravity, hydrodynamics and transport with full opacities (GR-FullOp, plotted in black); Newtonian gravity with full opacities and $\mathscr{O}(v / c)$ hydrodynamics and transport (N-FullOp, plotted in red); Newtonian gravity with reduced opacities and $\mathscr{O}(v / c)$ hydrodynamics and transport (N-ReducOp, plotted in green); and Newtonian gravity with $\mathscr{O}(v / c)$ hydrodynamics and reduced opacities, and $\mathscr{O}(1)$ transport (N-ReducOp-NOC, plotted in blue). The panels are: radial velocity (upper left), density (upper center), entropy (upper right), temperature ( $k T$, lower left), net electron fraction ( $\mathrm{Y}_{e}$, lower center, solid lines), net lepton fraction $\left(Y_{L}=\mathrm{Y}_{e}+\left(n_{v_{e}}-n_{\bar{v}_{e}}\right) / n_{\text {baryons }}\right.$, lower center, dashed lines), and pressure (lower right). 

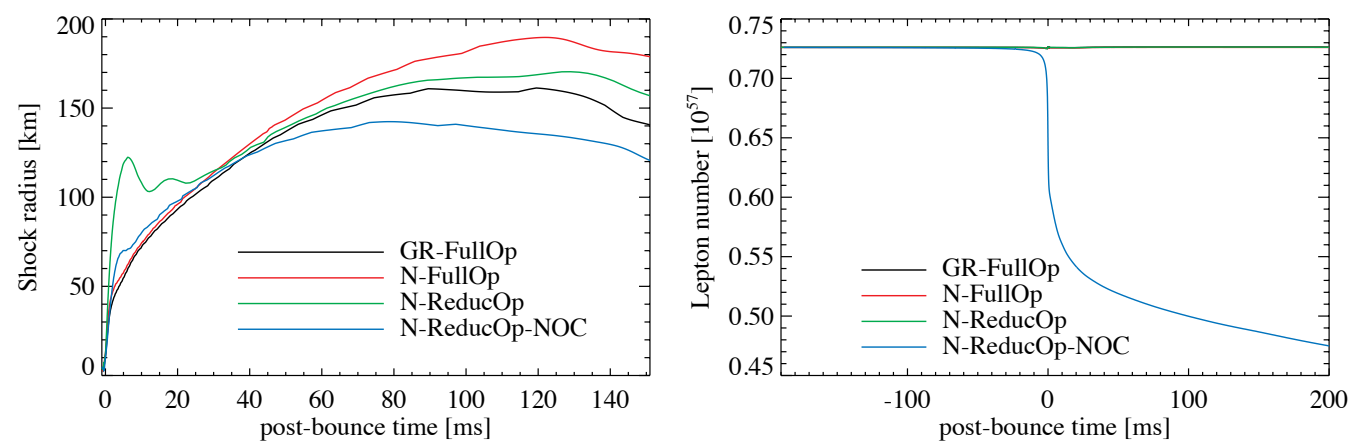

Figure 5: Shock trajectories in $\mathrm{km}$ (left) and conserved lepton number (right), versus time after bounce, for all models. The colors have the same meaning as in Figure 6 .

\subsubsection{General Relativity}

The first test examines the difference between models which include (GR-FullOp) or omit (NFullOp, red lines) general relativity in both the hydrodynamics and transport. At bounce (Figure $\mathbb{\text { G }}$ ), the deeper gravitational well of the GR-FullOp model results in a more compact core. After bounce, the GR model's deeper gravitational well and more compact PNS result in a smaller gain region that inhibits heating and results in a smaller shock radius relative to the Newtonian equivalent $[30,74,75]$. The more compact PNS also leads to a larger accretion luminosity [75] and higher mean energy, $\left\langle E_{v}\right\rangle_{\text {RMS }}$ for all species of neutrinos, especially the $v_{e}$ and $\bar{v}_{e}$ that drive the primary heating that could revive the shock. On the basis of 1D models, Liebendörfer et al. [74] suggested that once the multi-dimensional nature of the mechanism lengthened the time matter spent in the gain region, the higher luminosity and harder spectrum in GR models would result in stronger or more viable explosions. This has been recently confirmed by Mueller et al. [76], whose GR models explode when their Newtonian and approximate GR equilivents do not. Of the multidimensional codes mentioned in Sect. \, VERTEX [76] now uses a GR formulation, CHIMERA [77] and previously VERTEX [30, 78] use approximate GR, while Vulcan/2D [45, 79] and Zeus+IDSA [44] are Newtonian.
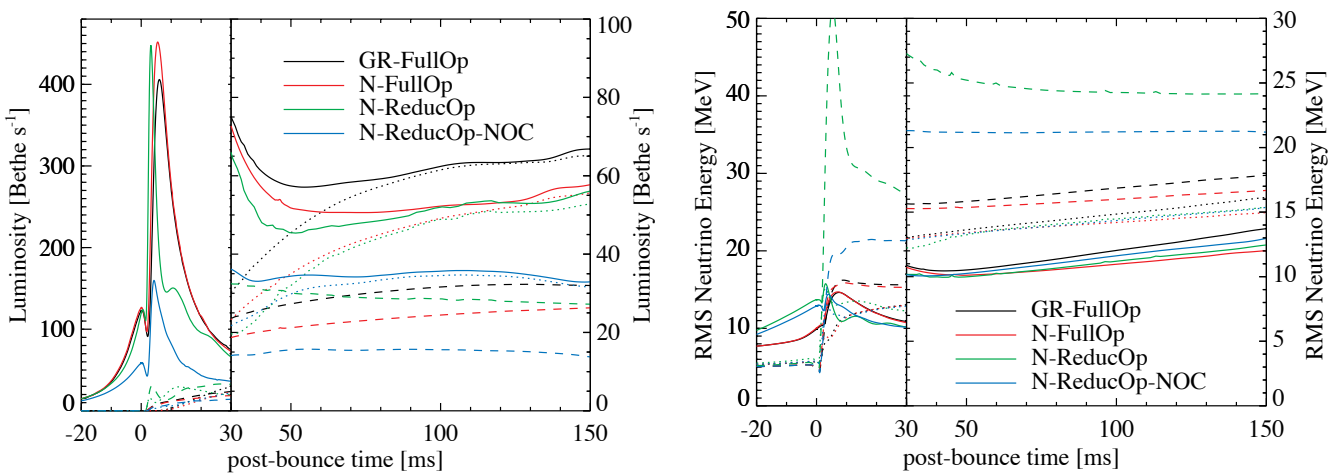

Figure 6: Comoving-frame neutrino luminosities and neutrino RMS energies, $\left\langle E_{v}\right\rangle_{\mathrm{RMS}}=$ $\left(\int d \mu d E E^{4} F / \int d \mu d E E^{2} F\right)^{1 / 2}$, measured at $400 \mathrm{~km}$ for all models. Colors are as in Figure 田. Electron neutrino, $v_{e}$, quantities are represented by solid lines, $\bar{v}_{e}$-quantities by dotted lines, and $v_{\mu \tau}$-quantities by dashed lines. $\bar{v}_{\mu \tau}$-quantities are indistinguishable from $v_{\mu \tau}$-quantities, and omitted from this figure. The luminosities are in Bethe $\mathrm{s}^{-1}$, where 1 Bethe $=10^{51}$ ergs. 


\subsubsection{Neutrino opacities}

Electron (positron) capture on nuclei in the collapsing core and on free protons (neutrons) in the shocked core are the dominant sources of cooling and deleptonization by emission and heating through the inverse absorption processes. Though scattering on the nucleons is the dominant source of total opacity and therefore controls the trapping of neutrinos, scattering on electrons preferentially scatters neutrinos to lower energies where the total opacity is lower and allows more neutrinos to escape during collapse [80].

To demonstrate the broad effects of modern opacities, the FullOp opacities used in the first two models (emission, absorption, and scattering on free nucleons [81]; isoenergetic scattering on $\alpha$-particles and heavy nuclei [63]; scattering of neutrinos on electrons (NES) and positrons (NPS) [82]; production of neutrino pairs from $e^{+} e^{-}$annihilation [82] and nucleon-nucleon bremsstrahlung [83]; and electron capture (EC) on nuclei using the LMSH EC table $[64,68]$ ) are simplified to ReducOp. In ReducOp the non-isoenergetic scattering (NIS) on nucleons is replaced by isoenergetic scattering (IS) [63] as are nucleon emission and scattering rates. To remove the rest of the NIS opacities, NES and NPS were both omitted entirely as their total opacity is small and primarily contribute through energy downscattering. Finally, the LMSH EC table was replaced by IPM nuclear EC [63] to complete the simplified ReducOp opacity set. The broad effect of this change in opacity can be seen in the difference between the N-FullOp (red) model and N-ReducOp (green) models in Figures $\mathbf{A}$ 6. Reduced electron capture and deleptonization from the simplified EC and the lack of NIS energy downscattering leads to higher core $\mathrm{Y}_{e}$ and $Y_{L}=\mathrm{Y}_{e}+Y_{v}$. The larger number of trapped neutrinos $Y_{V}$ in N-ReducOp is evident in the center lower panel of Figure $⿴ 囗$ 田 as the large gap between $\mathrm{Y}_{e}$ (solid lines) and $Y_{L}$ (dashed lines). The higher core $Y_{L}$ leads to the formation of the shock at a larger mass coordinate (shallower in the gravitational well) and a vigorous initial shock with "ringing" (Figure [5). The IPM EC not only effective removes all electron capture at high densities, but overestimates the EC at lower densities. This leads to more rapid collapse and higher density in the outer core (see near $0.9 M_{\odot}$ in Figure $⿴$ ) but also a steeper density gradient that slows down shock expansion. These reduced opacities together result in a lower shock trajectory that is less favorable to explosion. Of the multidimensional codes, CHIMERA and VERTEX use opacities similar to FullOp, Vulcan/2D and Zeus+IDSA rely on numerical methods that do not couple energies in the collision integral (no NIS, IS only) and therefore use opacities that are similar to ReducOp with Zeus+IDSA omitting $v_{\mu \tau}$ completely.

\subsubsection{Observer corrections}

In solving the transport equation we have a choice of both coordinates and frame. The Lagrangian coordinates moving with the fluid are natural for 1D and particle-based codes, while fixed Eulerian coordinates are natural for multidimensional grid-based codes. For the frame we may choose a frame locally co-moving with fluid, which is the frame where the radiation-matter interactions are defined but requiring numerous velocity-dependent terms on the left-hand side of the transport equation (see Eq. W. D) ; or we may choose the external observer (or lab) frame in which there are no velocity-dependent terms on the 1.h.s., but the interactions that compose the collision integral must be transformed from their natural co-moving frame. Including the effects of fluid motion and GR in the transport of photons or neutrinos requires either a transformation of the collision 
term or the l.h.s. of the transport equation. To better understand the observer corrections we start with the co-moving form ( $E_{0}$ and $\mu_{0}$ as the energy and cosine of the neutrino propagation angle) of the $\mathscr{O}(v)$ spherical transport equation for the invariant neutrino distribution function, $f$, in Eulerian coordinates $(r, \tilde{t})$ :

$$
\begin{aligned}
& \left(1+\mu_{0} v\right) \frac{\partial f}{\partial \tilde{t}}+\left(\mu_{0}+v\right) \frac{\partial f}{\partial r}+\frac{1-\mu_{0}^{2}}{r} \frac{\partial f}{\partial \mu_{0}} \\
& \quad+\left(\frac{v}{r}-\frac{\partial v}{\partial r}\right) \mu_{0}\left(1-\mu_{0}^{2}\right) \frac{\partial f}{\partial \mu_{0}}+\left[\mu_{0}^{2}\left(\frac{v}{r}-\frac{\partial v}{\partial r}\right)-\frac{v}{r}\right] E_{0} \frac{\partial f}{\partial E_{0}}=\frac{1}{E_{0}} C[f] .
\end{aligned}
$$

The velocity-dependent terms with the $E_{0}$ and $\mu_{0}$ derivatives include corrections to the energy and angle due to observer motion and we classify these terms as "observer corrections." When removed, the transport equation becomes much simpler,

$$
\left(1+\mu_{0} v\right) \frac{\partial f}{\partial \tilde{t}}+\left(\mu_{0}+v\right) \frac{\partial f}{\partial r}+\frac{1-\mu_{0}^{2}}{r} \frac{\partial f}{\partial \mu_{0}}=\frac{1}{E_{0}} C[f]
$$

lacking the coupling of energy groups required by the observer corrections and more closely resembling the lab-frame formulation. (It is not a lab-frame formulation as that would require a Lorentz-transformed lab-frame collision integral, $C^{\mathrm{Lab}}[f]$.)

Agile-BOLTZTRAN uses $F=f / \rho$ for the transport variable in Lagrangian coordinates $(t, m)$, so the "no observer corrections" transport equation (Eq. B.2) must be translated into an appropriate form:

$$
\frac{\partial F}{\partial t}+4 \pi \mu_{0} \frac{\partial\left(r^{2} \rho F\right)}{\partial m}+\frac{1}{r} \frac{\partial\left[\left(1-\mu_{0}^{2}\right) F\right]}{\partial \mu_{0}}+\frac{F}{\rho} \frac{\partial \rho}{\partial t}=\frac{1}{E_{0}} C[F] .
$$

If we multiply Eq 3.3 by $E_{0}{ }^{2}$ and a constant and integrate over $d E_{0}$ and $d \mu_{0}$, the $\partial / \partial \mu_{0}$ term will vanish and we have an expression for the evolution of neutrino number. We can again integrate over the mass of the entire model and the $\partial / \partial m$ term will reduce to a surface flux. Integration of the collision integral over mass gives the total lepton conversion rate $\left(v_{e} \leftrightarrow e^{-}\right.$, etc.), which should balance with the integrated total change in neutrino number from the integrated time derivative term plus the surface flux. However, in this form we can see that this is not the case, there is a "compression" term, $(F / \rho)(\partial \rho / \partial t)$, which does not integrate away. This term is part of the terms remaining in Eq. B.2 after the observer corrections are removed with a counterpart "hidden" in the observer corrections we eliminated. One consequence of this "compression" term is that when the fluid containing neutrinos is compressed neutrinos will be artificially destroyed, and when the fluid expands neutrinos will be artificially created.

To consider the effect of ignoring the observer corrections, we compare the N-ReducOp model (green) with the N-ReducOp-NOC model (blue) that uses the "no observer corrections" transport

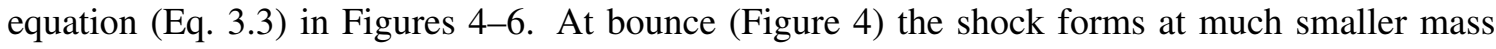
without observer corrections (coincidentally matching the most complete GR-FullOp model) due to the sharp reduction in $\mathrm{Y}_{e}$ and $Y_{L}$ to their lowest values at bounce. This is most starkly seen in the change from the largest core $Y_{V}$ (gap between dashed and solid lines in center lower panel of Figure 4 ) for the N-ReducOp model to the smallest $Y_{v}$ for N-ReducOp-NOC. This reduction in the $Y_{V}$ is a direct consequence of the "compression" term in Eq. 3.3], which we can also see in the conserved lepton number shown in Figure 5 (right panel). The conserved lepton number is a integration over the entire model for net electron and electron neutrino number including the appropriate 
boundary flux term. The first three models show excellent total lepton conservation over the entire simulation, but the N-ReducOp-NOC model drops slowly just before bounce and sharply during shock breakout with continued loss during the post-bounce accretion phase. This non-conservation demonstrates the unphysical nature of Eq. 3.3 and likely causes the large reduction in peak $L_{v_{e}}$ and the $\sim 40 \%$ drop in neutrino luminosities during the accretion phase when multi-dimensional models should begin the shock revival process. The reduced luminosities likely reflect that some of the neutrinos are (non-physically) destroyed instead of escaping. There are other effects missing from the N-ReducOp-NOC model including Doppler shifts and angular aberration which are more difficult to extract. Unlike the GR effects, where 1D models with GR looked less favorable but had latent potential to explode more vigorously due to the larger and harder neutrino emissions, in the N-ReducOp-NOC model the $\left\langle E_{v}\right\rangle_{\text {RMS }}$ is only slightly larger and the luminosity is significantly lower indicating that, if anything, the unfavorable nature of the model without observer corrections will be even more unfavorable in multi-dimensional models, of which CHIMERA, VERTEX, and Zeus+IDSA include appropriate observer corrections and Vulcan/2D omits them.

\section{Nucleosynthesis}

Large overabundances of elements in the periodic table spanning from oxygen through nickel are observed in core-collapse supernovae and their remnants. This nucleosynthesis involves a large number of nuclear reactions, strong and electromagnetic reactions as well as weak nuclear reactions involving capture of electrons, positrons and neutrinos. Experimental measurement and theoretical calculation of these reaction rates is another important connection between nuclear physics and astrophysics.

Observations of nuclear abundances allow nucleosynthesis calculations to place powerful constraints on conditions deep in the interior of supernovae and their progenitors, places hidden from direct observation. Unfortunately, the frequent failure over the past 40 years of self-consistent models to produce explosions has resulted in the reliance of core-collapse supernova nucleosynthesis studies on parameterized models, which replace the inner workings of the supernova with a kinetic energy piston [see, e.g., 84-87] or a thermal energy bomb [see, e.g. 88-90]. These two methods are largely compatible, with the largest differences coming in the inner regions of the ejecta [91]. It is the nucleosynthesis in this inner region that can be strongly affected by the details of the explosion mechanism [92]. In the case of the neutrino reheating mechanism, these effects include interaction with the tremendous flux of neutrinos and the temporal delay in achieving the explosion. This provides strong motivation to merge modeling of the nucleosynthesis with modeling of the supernova central engine, a task now being undertaken by several groups.

\subsection{Explosive Nucleosynthesis}

In the innermost regions of the ejecta, the passage of the shock heats matter to temperatures where Nuclear Statistical Equilibrium (NSE) is dominated by free nucleons and $\alpha$ particles [see, e.g., 93, 94]. As a result, most of the iron-peak species synthesized in core-collapse supernovae result from $\alpha$-rich freezeout [95]. As matter expands outward, it cools, allowing the light nuclei to recombine into iron, nickel, and neighboring nuclei. In the case of $\alpha$-rich freezeout, this recombination is incomplete, leaving a significant fraction of the matter still in the form of free nucleons 
and $\alpha$ particles. The detailed composition of this ejecta depends on its neutronization, which is set in the inner regions of the ejecta by neutrino interactions. Spherically symmetric models [e.g., $84,86,88$ ] find that above the innermost $\alpha$, iron, and nickel dominated regions, passage of the shock leaves a layer rich in the $\alpha$ isotopes: ${ }^{40} \mathrm{Ca},{ }^{36} \mathrm{Ar},{ }^{32} \mathrm{~S}$, and ${ }^{28} \mathrm{Si}$ - the products of incomplete silicon burning. Above this is a layer of ${ }^{16} \mathrm{O}$, in the outer portions of which significant fractions of ${ }^{20} \mathrm{Ne},{ }^{24} \mathrm{Mg}$, and ${ }^{12} \mathrm{C}$ are found. Finally, above the ${ }^{16} \mathrm{O}$ layer we find the helium layer and hydrogen envelope, if they were not driven off as part of a stellar wind.

One common property exhibited by all recent 1- and 2-D simulations utilizing spectral neutrino transport [see, e.g., 30, 42, 43, 74, 78] is a decrease in the neutronization in the outer part of the neutrino heating region, due to neutrino interactions. This is a feature that the parameterized bomb/piston nucleosynthesis models discussed above cannot replicate because they ignore the neutrino reheating mechanism. The neutronization is important because Galactic Chemical Evolution calculations and the relative neutron-poverty of terrestrial iron and neighboring elements strongly limit the amount of neutronized material that may be ejected into the interstellar medium by corecollapse supernovae [96]. Hoffman et al. [97] placed a limit of $10^{-4} M_{\odot}$ on the typical amount of neutron-rich $\left(\mathrm{Y}_{e}<0.47\right)$ ejecta allowed from each core-collapse supernova. Past multidimensional models of core-collapse supernovae using gray (energy-integrated or -averaged, i.e., not spectral) neutrino transport that did produce explosions tended to greatly exceed this limit [see, e.g., 21, 31]. To compensate, modelers have been forced to invoke the fallback of a considerable amount of matter onto the neutron star, occurring on a time scale longer than was simulated [98].

Work by Fröhlich et al. [99, 100] and Pruet et al. [101, 102] indicates that neutrino-powered explosions using spectral neutrino transport result in nucleosynthesis products qualitatively different in composition from either the parameterized bomb/piston nucleosynthesis models or gray transport models of the core-collapse mechanism. In the models used by both Fröhlich et al. and Pruett et al., the neutrino physics was artificially tuned in order to drive explosions. Thus, these models are also parameterized, but the explosion is explicitly neutrino-driven and the ejecta are determined self-consistently by the radiation hydrodynamics. These models have shown that the inclusion of neutrino captures on the ejecta, which decrease the neutronization, remedies some defects in the predictions made by previous models of nucleosynthesis, all of which neglected this important piece of physics. These interactions remove the over-production of neutron-rich iron and nickel isotopes that have plagued parameterized bomb and piston models. These simulations [99, 101] also show enhanced production of $\mathrm{Sc}, \mathrm{Cu}$, and $\mathrm{Zn}$; elements which observations of metal-poor stars [see, e.g., 103] suggest are 3-10 times more abundant than previous models predicted. Moreover, Fröhlich et al. [100] revealed a significant neutrino-driven flow to proton-rich nuclei above $A=64$, which was also confirmed by Pruet et al. [102], leading to the discovery of the $v$ p-process and suggesting that the innermost ejecta of core-collapse supernovae may be the production site of the light p-process nuclei. The discovery of a potential new nucleosynthesis process adds to the nuclear data that the astrophysical community needs [see e.g. 104]. Given the importance of these findings, it is essential these studies now be conducted in the context of self-consistent, multidimensional models using spectral neutrino transport.

Convection and other hydrodynamic instabilities greatly complicate the picture developed from spherically symmetric models, destroying strict compositional layering in the progenitor. For example, Arnett, in collaboration with Meakin and others [105-107], has demonstrated that con- 
vection occurs in the oxygen shell even before the explosion. For two decades it has been known that Rayleigh-Taylor instabilities originate when the shock crosses the $\mathrm{Si} / \mathrm{O}$ and $(\mathrm{C}+\mathrm{O}) / \mathrm{He}$ boundaries [89, 108-111]. However, these instabilities do not mix nickel to sufficiently high velocities to account for observations of Supernova 1987A [see 112, and references therein] and other corecollapse supernovae. The implication is that gross asymmetries must be present in the core and be part of the explosion mechanism itself. However, few simulations to date have directly considered both the neutrino-driven mechanism and the impact of this multidimensional behavior on the nucleosynthesis. An exception is Kifonidis et al. [113, 114], who used a parameterized neutrino luminosity and temperature [similar to 21] coupled to an $\alpha$-network (linking the $14 \alpha$ nuclei from ${ }^{4} \mathrm{He}$ to ${ }^{60} \mathrm{Zn}$ ), while separately tracking the neutronization. These simulations showed significantly higher velocities for iron-rich clumps than previous models [108-110]. Similar three dimensional models have recently been calculated by Hammer et al. [115]. These simulations are hampered in their ability to study the nucleosynthesis in detail, since the initial asymmetric parameterized neutrino-driven explosion models from Scheck [116] [see also 117] did not consider nuclear burning, but Hammer et al. [115] do show that in 3D, metal-rich clumps suffer much less deceleration at the shell interfaces. This results in asymptotic velocities of metal-rich clumps that are 2-3 times larger than was found in 2D models, in better agreement with observations.

The complex fluid flow that results from convection and the SASI witnessed in multi-dimensional models makes separating the ejecta from material trapped in the proto-neutron star more difficult. In the parameterized spherically symmetric models that dominate our understanding of supernova nucleosynthesis, the mass cut, a Lagrangian coordinate defining the inner edge of the ejecta, is a parameter determined by comparison to limits on the ejected mass of ${ }^{56} \mathrm{Ni}$ and other constraints. As Figure $\mathbb{Z}$, (taken from a 2D simulation using our CHIMERA supernova code [42]) illustrates, convection and the SASI complicate this picture, with some parcels of material with the same ini-

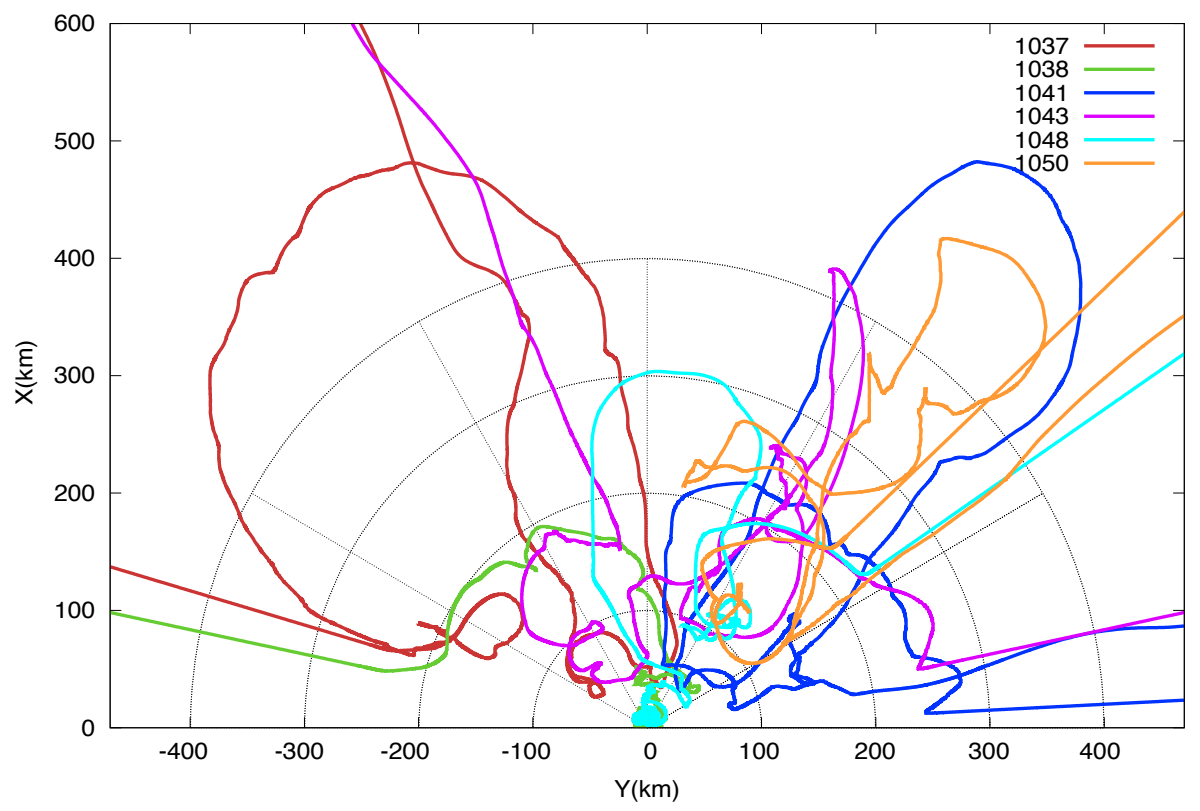

Figure 7: The time history of 6 example tracers from an initial spherically symmetric distribution within a $15 M_{\odot}$ star. Two of these tracers accrete onto the PNS while the other four are likely ejected after convecting through the neutrino heating region. 


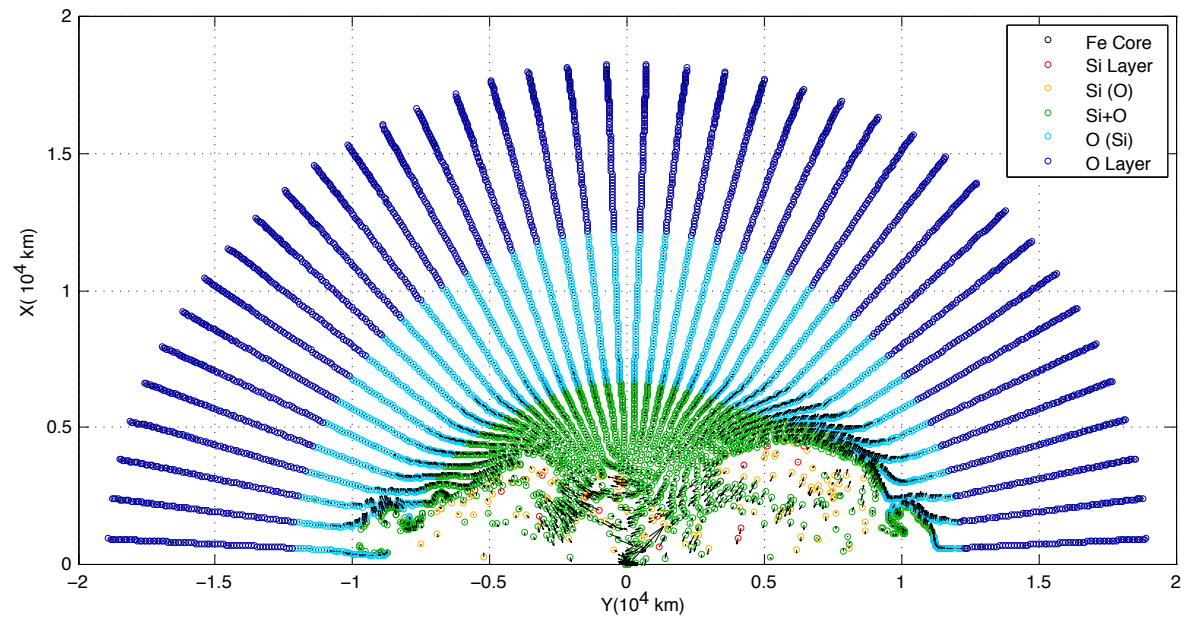

Figure 8: Tracer particles from a $25 M_{\odot}$ model at $\sim 1000 \mathrm{~ms}$ post-bounce have diverged from initial spherical symmetry illustrating that the definition of a "mass-cut" is not well defined in multi-dimensions.

tial radius being ejected while others are captured in the neutron star. Clearly in multi-dimensional models, and likely in Nature, the mass-cut loses its strictly spherical shape. It may even fail to be simply connected in the topological sense, with regions of eventual ejecta being immersed in material destined to form part of the neutron star. Limits on the neutron-richness of the iron and nickel ejecta in the neutrino-less parameterized nucleosynthesis models generally place the mass-cut in the oxygen layer. However, multi-dimensional simulations are beginning to reveal greater complexity. For example, Figure $\$$ (taken from another 2D CHIMERA simulation) shows that some of the matter that began deep in the silicon layer (red circles) of a 25 solar mass star seems likely to be ejected. Because of its exposure to the neutrino field, this matter is likely to be less neutron-rich than it was when the star began to collapse, easing the constraints on the neutron-richness of the ejecta.

As a result of their gray neutrino transport, the simulations of Kifonidis et al. [113] predict the ejection of much larger quantities of neutron-rich iron group elements than Galactic Chemical Evolution (GCE) seems to allow. In contrast, preliminary analysis of our spectral 2D CHIMERA results, based on analytic extension of tracer histories to late times [118, 119], document the development of the proton-rich ejecta and the $v$ p-process, though at a somewhat reduced level compared to previous work [100], because of faster expansion time scales. This highlights the critical dependence of the strength of the $v$ p-process on the expansion time scale. Recent extended simulations [120-122] highlight the impact (or lack thereof) of the forming reverse shock on the expansion time scale and hence the strength of the $v$ p-process, providing further justification for the merger of explosion mechanism models and nucleosynthesis models, as well as the extension of these models in time until the nucleosynthesis is complete. Such merged simulations are necessary to fully understand the quantity and distribution of intermediate-mass and iron-peak nuclei made in core-collapse supernovae, likely resolving the existing issue of too-neutron-rich ejecta, and helping us understand the role of massive stars in GCE. These simulations will also likely reveal the location and strength of the $v$ p-process, indicating the range of p-process species whose cosmic abundance also result from the deaths of massive stars. 


\section{2 r-Process}

Burbidge et al. [123] and Cameron [124] divided the observed abundance of the isotopes heavier than iron among three distinct processes. On the basis of the correlation between nuclear properties and cosmic abundances, we know that roughly half of these isotopes are the result of a slow neutron capture process, termed the s-process. Most of the remainder are the result of a rapid neutron capture process, the r-process, while an assortment of rarer, heavy proton-rich isotopes are ascribed to a p-process, originally thought to be driven by proton capture. Based on well-measured nuclear data from stable nuclei and mature models of stellar evolution, we have considerable confidence that the s-process occurs over thousands of years in the hydrogen- and helium-rich nuclear burning shells that sit atop the inert carbon-oxygen $(\mathrm{CO})$ cores in Asymptotic Giant Branch (AGB) stars. This confidence is buoyed by observations of red-giant stars with large over-abundances of s-process elements like barium and radioactive technetium (see [125] for a recent review). In the cases of the r-process and p-process, we do not have well-measured nuclear data for the highly radioactive isotopes involved, nor mature models of the potential astrophysical sites, nor direct observations.

Recovery of the observed pattern of r-process species requires a combination of high-entropy and neutron-rich material, acting on a time scale of seconds, with the exact balance varying between different proposed sites. The appearance of r-process elements in the spectra of old low-mass stars whose heavy element abundance are less than 1/1000th of the Sun (termed metal-poor stars) indicates that the formation of r-process elements began very early in Galactic Chemical Evolution, suggesting that the r-process occurs during the death throes of short-lived, massive stars [126]. The observation in earlier supernova models [e.g., 27] of neutron-rich matter in the high-entropy hot bubble that surrounds the PNS after the supernova explosion has been launched, naturally suggested this PNS wind as a potential site of the r-process [127]. Indeed, Woosley et al. [128] were able to produce a good match to the Solar System r-process pattern as the result of a neutron-rich, $\alpha$-rich freezeout. However, Fuller and Meyer [129, 130] pointed out that neutrino interactions can inhibit such a freezeout. Since the protons are tied up in $\alpha$-particles, which are relatively inert to neutrino interactions, neutrino capture on free neutrons, which produces protons, will destroy the neutron-richness of the matter, severely inhibiting the formation of the r-process. This problem is exacerbated in models which employ spectral neutrino transport, which give rise to proton-rich ejecta $[99,101]$. The electron fraction in the innermost hot bubble is set primarily by electron neutrino and electron antineutrino capture on free nucleons. For the matter to be neutron-rich, the difference in the electron neutrino and antineutrino spectra must overcome the mass difference between the neutron and the proton [131]. As a result of the inclusion of more types of neutrino opacity, all current simulations with spectral neutrino transport exhibit too small of a difference between the electron neutrino and antineutrino spectra. Thus, as the matter is ejected and the density drops, it becomes proton-rich once electron degeneracy is removed. The simulations used by Fröhlich et al. [99] and Pruet et al. [101] exhibit this proton-richness over the first $\sim 1$ second after core bounce. Recent, extended spherically symmetric simulations [132, 133] suggest that protonrich ejecta continue well into the PNS wind phase, suggesting iron core-collapse supernovae are an unlikely r-process site. However, 3D simulations with spectral transport, the first of which are currently underway, could improve the prospects for PNS-wind r-process nucleosynthesis because 
the properties of the wind are influenced by the continued accretion and by the development of the reverse shock. Additional physics, like active-sterile neutrino flavor transformations [134] or collective neutrino flavor transformations [135], could potentially revive the prospects of this r-process site, as well.

Other mechanisms can also fulfill the deduced correlation between the r-process and the deaths of massive stars. Surman et al. [136] have suggested that outflows from the accretion disk around the newly formed black hole in the collapsar scenario could be neutron-rich if the accretion rate is sufficiently high $\left(\sim 10 M_{\odot} s^{-1}\right)$. 3D simulations with spectral neutrino transport over the next 5 years will likely be able for the first time to simulate the formation and evolution of this accretion disk beginning at the onset of core collapse, putting this model on as firm a computational footing as conventional core-collapse supernova models and answering whether neutron-rich ejecta truly result from self-consistent collapsar simulations that begin when stellar evolution ends.

Ning et al. [137] have suggested a mechanism wherein the r-process can be produced in a supernova arising from an $\mathrm{ONeMg}$ core, whose progenitors are 8-11 $M_{\odot}$ stars. Unlike the iron core case, the collapsing core in these stars is surrounded by low-density helium and hydrogen layers (in contrast to the much-higher-density silicon and oxygen layers present in a more massive star) producing a sharp density gradient at the surface of the stellar core. Once the shock, reinvigorated by neutrino heating, reaches this gradient, it accelerates. In the model of Ning et al. [137], the fast expansion of the shocked matter in the weakly neutron-rich surface layers of the ONeMg core leads to an r-process. Unfortunately, the best simulations of this process to date $[138,139]$ do not produce the required combination of temperature, entropy, and expansion time scale for currently available stellar progenitors. Most recently, Wanajo et al. [140] have shown that 2D simulations can eject moderately neutron-rich convective "lumps" capable of forging elements up to $\mathrm{N}=50$. They raise the possibility that $3 \mathrm{D}$ simulations and/or improved resolution could result in tiny amounts of matter that are neutron-rich enough to produce at least the lower-mass r-process species. Here too, it is likely that in the next 5 years, for the first time, this exciting possibility can be explored in 3D simulations with all the required physics, including spectral neutrino transport.

\section{Summary}

Advancement in our understanding of core-collapse supernovae, both the mechanism that drives the explosion and the nucleosynthesis that results, is driven simultaneously by improvements in our ability to model macroscopic phenomena like hydrodynamics and radiation transport with greater physical fidelity and improvements in our understanding of the microscopic physics that drives these events. Improvements in computational technology and our ability to harness it, are for the first time allowing three dimensional models with all the necessary physics. Matching this greatly improved physical fidelity at macroscopic scales requires continued improvement of the physics at microscopic scales. Much of this microscopic physics, for example, the nuclear equation of state and nuclear reactions of interest to both nucleosynthesis and the collapse of the stellar core, requires an understanding of the physics of atomic nuclei. Here too, improvements in computational technology and our ability to harness it, constrained where possible by experimental measurements, are improving our understanding. 


\section{References}

[1] T. Preibisch and H. Zinnecker, AJ 117, 2381 (1999).

[2] J. P. Phillips, G. Ramos-Larios, and J. A. Perez-Grana, MNRAS 397, 1215 (2009).

[3] E. Cappellaro and M. Turatto, in The Influence of Binaries on Stellar Population Studies, edited by D. Vanbeveren (2001), Astrophysics and Space Science Library vol. 264, p. 199.

[4] A. V. Filippenko, ARA\&A 35, 309 (1997).

[5] K. Nomoto, F.-K. Thielemann, and K. Yokoi, ApJ 286, 644 (1984).

[6] W. Hillebrandt and J. C. Niemeyer, ARA\&A 38, 191 (2000).

[7] A. M. Khokhlov, A\&A 245, 114 (1991).

[8] T. Plewa, A. C. Calder, and D. Q. Lamb, ApJ 612, L37 (2004).

[9] I. Iben, Jr. and A. Renzini, ARA\&A 21, 271 (1983).

[10] A. Bressan, F. Fagotto, G. Bertelli, and C. Chiosi, A\&AS 100, 647 (1993).

[11] S. E. Woosley, A. Heger, and T. A. Weaver, Rev. Mod. Phys. 74, 1015 (2002).

[12] A. I. MacFadyen, S. E. Woosley, and A. Heger, ApJ 550, 410 (2001).

[13] C. Ritossa, E. García-Berro, and I. Iben, Jr., ApJ 515, 381 (1999).

[14] I. Iben, Jr., C. Ritossa, and E. García-Berro, ApJ 489, 772 (1997).

[15] S. Miyaji and K. Nomoto, ApJ 318, 307 (1987).

[16] H.-T. Janka, K. Langanke, A. Marek, G. Martínez-Pinedo, and B. Müller, Phys. Rep. 442, 38 (2007).

[17] A. Mezzacappa, Annu. Rev. Nucl. Part. Sci. 55, 467 (2005).

[18] J. R. Wilson, in Numerical Astrophysics, edited by J. M. Centrella, J. M. LeBlanc, and R. L. Bowers (Jones and Bartlett, Boston, 1985), pp. 422-434.

[19] H. A. Bethe and J. R. Wilson, ApJ 295, 14 (1985).

[20] A. Burrows and J. Goshy, ApJ 416, L75 (1993).

[21] H.-T. Janka and E. Müller, A\&A 306, 167 (1996).

[22] A. Mezzacappa, A. C. Calder, S. W. Bruenn, J. M. Blondin, M. W. Guidry, M. R. Strayer, and A. S. Umar, ApJ 495, 911 (1998).

[23] H.-T. Janka, A\&A 368, 527 (2001). 
[24] O. E. B. Messer, A. Mezzacappa, S. W. Bruenn, and M. W. Guidry, ApJ 507, 353 (1998).

[25] A. Mezzacappa, M. Liebendörfer, O. E. Messer, W. R. Hix, F.-K. Thielemann, and S. W. Bruenn, Phys. Rev. Lett. 86, 1935 (2001).

[26] L. Smarr, J. R. Wilson, R. T. Barton, and R. L. Bowers, ApJ 246, 515 (1981).

[27] J. R. Wilson and R. W. Mayle, Phys. Rep. 227, 97 (1993).

[28] J. A. Miralles, J. A. Pons, and V. A. Urpin, ApJ 574, 356 (2002).

[29] S. W. Bruenn, E. A. Raley, and A. Mezzacappa, ArXiv e-prints astro-ph/0404099 (2004).

[30] R. Buras, M. Rampp, H.-T. Janka, and K. Kifonidis, A\&A 447, 1049 (2006).

[31] M. Herant, W. Benz, W. R. Hix, C. L. Fryer, and S. A. Colgate, ApJ 435, 339 (1994).

[32] A. Burrows, J. Hayes, and B. A. Fryxell, ApJ 450, 830 (1995).

[33] C. L. Fryer and M. S. Warren, ApJ 601, 391 (2004).

[34] S. W. Bruenn, C. J. Dirk, A. Mezzacappa, J. C. Hayes, J. M. Blondin, W. R. Hix, and O. E. B. Messer, J. Phys.: Conf. Ser. 46, 393 (2006).

[35] J. Blondin, A. Mezzacappa, and C. DeMarino, ApJ 584, 971 (2003).

[36] H.-T. Janka, R. Buras, F. S. Kitaura Joyanes, A. Marek, M. Rampp, and L. Scheck, Nucl. Phys. A 758, 19 (2005).

[37] A. Burrows, E. Livne, L. Dessart, C. D. Ott, and J. Murphy, ApJ 640, 878 (2006).

[38] N. Ohnishi, K. Kotake, and S. Yamada, ApJ 641, 1018 (2006).

[39] E. M. D. Symbalisty, ApJ 285, 729 (1984).

[40] S. Akiyama, J. C. Wheeler, D. L. Meier, and I. Lichtenstadt, ApJ 584, 954 (2003).

[41] A. Burrows, L. Dessart, E. Livne, C. D. Ott, and J. Murphy, ApJ 664, 416 (2007).

[42] S. W. Bruenn, A. Mezzacappa, W. R. Hix, J. M. Blondin, P. Marronetti, O. E. B. Messer, C. J. Dirk, and S. Yoshida, J. Phys.: Conf. Ser. 180, 012018 (2009).

[43] A. Marek and H.-T. Janka, ApJ 694, 664 (2009).

[44] Y. Suwa, T. Takiwaki, S. C. Whitehouse, M. Liebendörfer, and K. Sato, PASP 62, L49 (2010).

[45] A. Burrows, E. Livne, L. Dessart, C. D. Ott, and J. Murphy, ApJ 655, 416 (2007).

[46] D. G. Ravenhall, C. J. Pethick, and J. R. Wilson, Phys. Rev. Lett. 50, 2066 (1983).

[47] J. Lattimer and F. D. Swesty, Nucl. Phys. A 535, 331 (1991). 
[48] D. Q. Lamb, J. M. Lattimer, C. J. Pethick, and D. G. Ravenhall, Phys. Rev. Lett. 41, 1623 (1978).

[49] H. Shen, H. Toki, K. Oyamatsu, and K. Sumiyoshi, Prog. Theor. Phys. 100, 1013 (1998).

[50] K. Oyamatsu, Nucl. Phys. A 561, 431 (1993).

[51] J. R. Wilson and G. J. Mathews, Relativistic Numerical Hydrodynamics, Cambridge Monographs on Mathematical Physics (Cambridge University Press, Cambridge, 2003).

[52] R. L. Bowers and J. R. Wilson, ApJS 50, 115 (1982).

[53] R. W. Mayle and J. R. Wilson, in Supernovae, edited by S. E. Woosley (Springer, New York, 1991), p. 333.

[54] T. L. McAbee and J. R. Wilson, Nucl. Phys. A 576, 626 (1994).

[55] E. Baron, J. Cooperstein, and S. Kahana, Phys. Rev. Lett. 55, 126 (1985).

[56] H. Muther, M. Prakash, and T. L. Ainsworth, Phys. Lett. B 199, 469 (1987).

[57] B. Friedman, V. R. Pandharipande, and Q. N. Usmani, Nucl. Phys. A 372, 483 (1981).

[58] M. Hempel and J. Schaffner-Bielich, Nucl. Phys. A 837, 210 (2010).

[59] G. Shen, C. J. Horowitz, and S. Teige, Phys. Rev. C 83, 035802 (2011).

[60] G. Shen, C. J. Horowitz, and E. O’Connor, Phys. Rev. C 83, 065808 (2011).

[61] M. Liebendörfer, O. E. B. Messer, A. Mezzacappa, S. W. Bruenn, C. Y. Cardall, and F.-K. Thielemann, ApJS 150, 263 (2004).

[62] W. R. Hix, O. E. B. Messer, A. Mezzacappa, M. Liebendörfer, J. M. Sampaio, K. Langanke, D. J. Dean, and G. Martinez-Pinedo, Phys. Rev. Lett. 91, 201102 (2003).

[63] S. W. Bruenn, ApJS 58, 771 (1985).

[64] K. Langanke, G. Martínez-Pinedo, J. M. Sampaio, D. J. Dean, W. R. Hix, O. E. Messer, A. Mezzacappa, M. Liebendörfer, H.-T. Janka, and M. Rampp, Phys. Rev. Lett. 90, 241102 (2003).

[65] H. A. Bethe, G. E. Brown, J. Applegate, and J. M. Lattimer, Nucl. Phys. A 324, 487 (1979).

[66] G. M. Fuller, ApJ 252, 741 (1982).

[67] J. Cooperstein and J. Wambach, Nucl. Phys. A 420, 591 (1984).

[68] K. Langanke and G. Martínez-Pinedo, Nucl. Phys. A 673, 481 (2000).

[69] K. Langanke, E. Kolbe, and D. J. Dean, Phys. Rev. C 63, 032801 (2001).

[70] G. Martínez-Pinedo, M. Liebendörfer, and D. Frekers, Nucl. Phys. A 777, 395 (2006). 
[71] A. Juodagalvis, K. Langanke, W. R. Hix, G. Martínez-Pinedo, and J. M. Sampaio, Nucl. Phys. A 848, 454 (2010).

[72] E. J. Lentz, A. Mezzacappa, O. E. B. Messer, W. R. Hix, and S. W. Bruenn, ApJ (2012), submitted.

[73] E. J. Lentz, A. Mezzacappa, O. E. B. Messer, M. Liebendörfer, W. R. Hix, and S. W. Bruenn, ApJ 747, 73 (2012).

[74] M. Liebendörfer, A. Mezzacappa, F.-K. Thielemann, O. E. B. Messer, W. R. Hix, and S. W. Bruenn, Phys. Rev. D 63, 103004 (2001).

[75] S. W. Bruenn, K. R. De Nisco, and A. Mezzacappa, ApJ 560, 326 (2001).

[76] B. Mueller, H.-T. Janka, and A. Marek, ArXiv e-prints (2012), .

[77] S. W. Bruenn et al., ApJS , in preparation (2012).

[78] M. Rampp and H.-T. Janka, A\&A 396, 361 (2002).

[79] E. Livne, A. Burrows, R. Walder, I. Lichtenstadt, and T. A. Thompson, ApJ 609, 277 (2004).

[80] A. Mezzacappa and S. W. Bruenn, ApJ 410, 740 (1993).

[81] S. Reddy, M. Prakash, J. M. Lattimer, and J. A. Pons, Phys. Rev. C 59, 2888 (1999).

[82] P. J. Schinder and S. L. Shapiro, ApJS 50, 23 (1982).

[83] S. Hannestad and G. Raffelt, ApJ 507, 339 (1998).

[84] S. E. Woosley and T. A. Weaver, ApJS 101, 181 (1995).

[85] T. Rauscher, A. Heger, R. D. Hoffman, and S. E. Woosley, ApJ 576, 323 (2002).

[86] M. Limongi and A. Chieffi, ApJ 592, 404 (2003).

[87] S. E. Woosley and A. Heger, Phys. Rep. 442, 269 (2007).

[88] F.-K. Thielemann, K. Nomoto, and M. Hashimoto, ApJ 460, 408 (1996).

[89] S. Nagataki, T. M. Shimizu, and K. Sato, ApJ 495, 413 (1998).

[90] H. Umeda and K. Nomoto, ApJ 673, 1014 (2008).

[91] M. B. Aufderheide, E. Baron, and F. K. Thielemann, ApJ 370, 630 (1991).

[92] C. Fryer, P. Young, M. E. Bennet, S. Diehl, F. Herwig, R. Hirschi, A. Hungerford, M. Pignatari, G. Magkotsios, G. Rockefeller, et al., in Proceedings of Nuclei in the Cosmos X, edited by H. Schatz and et al. (SISSA Proceedings of Science, 2008), p. 101.

[93] F. E. Clifford and R. J. Tayler, Mem. RAS 69, 21 (1965). 
[94] D. Hartmann, S. E. Woosley, and M. F. El Eid, ApJ 297, 837 (1985).

[95] S. E. Woosley, W. D. Arnett, and D. D. Clayton, ApJS 26, 231 (1973).

[96] V. Trimble, A\&AR 3, 1 (1991).

[97] R. D. Hoffman, S. E. Woosley, G. M. Fuller, and B. S. Meyer, ApJ 460, 478 (1996).

[98] C. L. Fryer and A. Hungerford, in The r-Process: The Astrophysical Origin of the Heavy Elements and Related Rare Isotope Accelerator Physics (2004), p. 234.

[99] C. Fröhlich, P. Hauser, M. Liebendörfer, G. Martínez-Pinedo, F.-K. Thielemann, E. Bravo, N. T. Zinner, W. R. Hix, K. Langanke, A. Mezzacappa, et al., ApJ 637, 415 (2006).

[100] C. Fröhlich, G. Martínez-Pinedo, M. Liebendörfer, F. K. Thielemann, E. Bravo, W. R. Hix, K. Langanke, and N. T. Zinner, Phys. Rev. Lett. 96, 142502 (2006).

[101] J. Pruet, S. E. Woosley, R. Buras, H.-T. Janka, and R. D. Hoffman, ApJ 623, 325 (2005).

[102] J. Pruet, R. D. Hoffman, S. E. Woosley, H.-T. Janka, and R. Buras, ApJ 644, 1028 (2006)

[103] R. Cayrel, E. Depagne, M. Spite, V. Hill, F. Spite, P. François, B. Plez, T. Beers, F. Primas, J. Andersen, et al., A\&A 416, 1117 (2004).

[104] C. Weber, V.-V. Elomaa, R. Ferrer, C. Fröhlich, D. Ackermann, J. Äystö, G. Audi, L. Batist, K. Blaum, M. Block, et al., Phys. Rev. C 78, 054310 (2008).

[105] C. A. Meakin and D. Arnett, ApJ 637, L53 (2006).

[106] G. Bazan and D. Arnett, ApJ 496, 316 (1998).

[107] S. M. Asida and D. Arnett, ApJ 545, 435 (2000).

[108] I. Hachisu, T. Matsuda, K. Nomoto, and T. Shigeyama, ApJ 358, L57 (1990).

[109] E. Müller, B. Fryxell, and D. Arnett, A\&A 251, 505 (1991).

[110] M. Herant and W. Benz, ApJ 387, 294 (1992).

[111] J. Kane, D. Arnett, B. A. Remington, S. G. Glendinning, G. Bazán, E. Müller, B. A. Fryxell, and R. Teyssier, ApJ 528, 989 (2000).

[112] R. McCray, ARA\&A 31, 175 (1993).

[113] K. Kifonidis, T. Plewa, L. Scheck, H.-T. Janka, and E. Müller, A\&A 453, 661 (2006).

[114] K. Kifonidis, T. Plewa, H.-T. Janka, and E. Müller, A\&A 408, 621 (2003).

[115] N. J. Hammer, H.-T. Janka, and E. Müller, ApJ 714, 1371 (2010).

[116] L. Scheck, Ph.D. thesis, Technical University Munich (2007). 
[117] L. Scheck, K. Kifonidis, H.-T. Janka, and E. Müller, A\&A 457, 963 (2006).

[118] C.-T. Lee, Ph.D. thesis, University of Tennessee (2008).

[119] O. E. B. Messer, S. W. Bruenn, J. M. Blondin, M. A. Chertkow, W. R. Hix, C. Lee, E. J. Lentz, P. Marronetti, and K. N. Yakunin, in Proceedings of Nuclei in the Cosmos XI, edited by G. Martínez-Pinedo and et al. (SISSA Proceedings of Science, 2010), p. 027.

[120] S. Wanajo, H.-T. Janka, and S. Kubono, ApJ 729, 46 (2011).

[121] A. Arcones and H.-T. Janka, A\&A 526, A160 (2011).

[122] L. F. Roberts, S. E. Woosley, and R. D. Hoffman, ApJ 722, 954 (2010), .

[123] E. M. Burbidge, G. R. Burbidge, W. A. Fowler, and F. Hoyle, Rev. Mod. Phys. 29, 547 (1957).

[124] A. G. W. Cameron, PASP 69, 201 (1957), atomic Energy of Canada, Ltd., CRL-41.

[125] F. Käppeler, R. Gallino, S. Bisterzo, and W. Aoki, Rev. Mod. Phys. 83, 157 (2011).

[126] D. Argast, M. Samland, F.-K. Thielemann, and Y.-Z. Qian, A\&A 416, 997 (2004).

[127] B. S. Meyer, G. J. Mathews, W. M. Howard, S. E. Woosley, and R. D. Hoffman, ApJ 399, 656 (1992).

[128] S. E. Woosley, J. R. Wilson, G. J. Mathews, R. D. Hoffman, and B. S. Meyer, ApJ 433, 229 (1994).

[129] G. M. Fuller and B. S. Meyer, ApJ 453, 792 (1995).

[130] B. S. Meyer, G. C. McLaughlin, and G. M. Fuller, Phys. Rev. C 58, 3696 (1998).

[131] Y.-Z. Qian and S. E. Woosley, ApJ 471, 331 (1996).

[132] T. Fischer, S. C. Whitehouse, A. Mezzacappa, F.-K. Thielemann, and M. Liebendörfer, A\&A 517, A80 (2010).

[133] L. Hüdepohl, B. Müller, H.-T. Janka, A. Marek, and G. G. Raffelt, Phys. Rev. Lett. 104, 251101 (2010).

[134] J. Beun, G. C. McLaughlin, R. Surman, and W. R. Hix, Phys. Rev. D 73, 093007 (2006).

[135] H. Duan, A. Friedland, G. C. McLaughlin, and R. Surman, J. Phys. G: Nucl. Phys. 38, 035201 (2011).

[136] R. Surman, G. C. McLaughlin, and W. R. Hix, ApJ 643, 1057 (2006).

[137] H. Ning, Y.-Z. Qian, and B. S. Meyer, ApJ 667, L159 (2007), .

[138] H.-T. Janka, B. Müller, F. S. Kitaura, and R. Buras, A\&A 485, 199 (2008).

[139] F. S. Kitaura, H.-T. Janka, and W. Hillebrandt, A\&A 450, 345 (2006).

[140] S. Wanajo, H.-T. Janka, and B. Müller, ApJ 726, L15 (2011). 